CONCURSO ESTUDIANTIL DE DISEÑO

\title{
Kui xékua: Ayuda para adultos mayores
}

\author{
Diego A. L. de Guevara Heras, Gilberto López \\ Antonio, Francisco J. Valencia Martínez, Maria del \\ Rosario Peralta Calvo
}

Publicado: 21 Septiembre 2016

\begin{abstract}
Resumen
Kui Xékua es un servicio diseñado para ayudar a los adultos mayores (AM) con el objetivo de mejorar la calidad de vida, coadyuvando en la realización de sus actividades cotidianas. Los AM aun cuando por su edad ven reducidas sus capacidades físicas para realizar trabajos pesados, para el traslado y movilidad de un lugar a otro, necesitan de manera significativa seguir incluyéndolos en la vida laboral, y así sentirse socialmente activos. Para lograr esto, Kui Xékua ofrece a los AM un brazalete que portarán para solicitar un tipo de ayuda, este se sincroniza con una aplicación web, gestionado por un administrador, quien atenderá las solicitudes, enviando a personal capacitado a la localización del AM y otorgar la ayuda. La usabilidad del brazalete del AM es relevante para que el servicio Kui Xékua, logre el objetivo planteado. En el presente trabajo se da a conocer la propuesta del servicio, justificando su desarrollo con la metodología Diseño Centrado en el Usuario.
\end{abstract}

Palabras clave: Diseño centrado en el usuario; estudio contextual; adultos mayores; aplicación web; brazalete; pruebas de Mago de OZ.

\section{Introducción}

En México, el número de AM está creciendo rápidamente, se estima que, en el año 2050, uno de cuatro mexicanos sea un adulto mayor. Esto es preocupante porque los AM tienen muchas necesidades particulares, tales como, atención médica, movilidad o socialización. Un gran número de adultos mayores no usan ningún tipo de tecnología (incluyendo teléfonos móviles) ya sea, porque no tienen acceso a ella o son poco adecuadas para que ellos las utilicen [1]. En la actualidad el estado de Oaxaca cuenta con 491,259 AM. La población de Teotitlán de Flores Magón cuenta con 8,969 habitantes, de cuya población el $10.4 \%$ son adultos mayores de 60 años en adelante, es decir, 932 personas. [2][3].

Con base en un estudio contextual realizado en Teotitlán de Flores Magón, se obtuvo que la mayor parte de adultos mayores

\footnotetext{
De Guevara Heras, DAL., López Antonio, G., Valencia Martínez, FJ., Peralta Calvo, MR.

Universidad de la Cañada

Km. 1.7 Carretera Teotitlán-Nanahuatipán,

Teotitlán de Flores Magón, Oaxaca

Email: li_diegoguevara@unca.edu.mx, li_gilbertolpz@unca.edu.mx,

li_franciscomtz@unca.edu.mx,mperalta@unca.edu.mx
}

presentan problemas para realizar alguna actividad que requiera trabajo físico rigoroso. Además, en su mayoría, este tipo de personas no cuentan con alguien que le pueda servir de apoyo en las actividades que ellos no pueden realizar. Otro resultado relevante de dicho estudio, es que la mayor parte de los adultos mayores han interactuado con teléfonos convencionales, lo que implica que mantienen una relación con la tecnología. Lo anterior permitió desarrollar una propuesta que consiste en un servicio que pueda apoyar a los adultos mayores en su vida cotidiana, incluyendo la tecnología y el apoyo de otras personas que tengan la disposición de ayudar a este sector vulnerable de la población. Debido a que dicha propuesta se centra en los AM, en las siguientes secciones se justifica el desarrollo bajo la metodología Diseño Centrado en el Usuario con la finalidad de que la solución propuesta cubra las necesidades detectadas en el estudio y que al incluir la tecnología coadyuve en la mejora de su calidad de vida.

\section{Solución propuesta}

La propuesta que se presenta se enfoca en implantar un servicio en la localidad, el cual se encargará de brindar ayuda a los AM, usando como herramientas: un wearable [4] de tipo brazalete, (ver Figura 1) mediante el cual los adultos mayores podrán pedir ayuda con tan sólo presionar un botón de este; y una aplicación web, (ver Figura 2) la cual se encargará de recibir las solicitudes de ayuda y dar seguimiento a las mismas asignando personal de apoyo que se encarguen de llegar al lugar donde fue solicitada la ayuda y realizar la actividad requerida. El brazalete está enfocado a los AM, mientras que la aplicación web está orientada a personas con experiencia en el manejo de las Tecnologías de la Información y Comunicación (TIC's). La razón del brazalete, es que se observó que para la realización de sus actividades, este no afectaría, al contrario puede ser más portable que un teléfono celular, por ejemplo.

El brazalete cuenta con elementos de retroalimentación para los AM, con la finalidad de hacer fácil y mejor entendimiento del uso de esta tecnología. El brazalete atiende tres categorías de ayuda, el usuario pulsará el botón que corresponda a la necesidad que tenga (botones 5, 6 y 7), esta solicitud será atendida desde la aplicación web, que es parte de la solución propuesta (más adelante se explicará con detalle). Cuando el AM haya pulsado un botón, se encenderá un led que le indicará que su solicitud está siendo atendida, además se le notificará por voz. El color de cada led ira cambiado según sea el estado en que se encuentre dicha solicitud: En camino (led en rojo), llego (led en naranja), 
terminado (led en verde). En este momento existe sincronía con la aplicación web. Véase la Figura 2.

La otra parte de la solución propuesta, es una aplicación web, que se encargará de atender todas las solicitudes de ayuda de los AM. Cuenta con dos secciones principales, en la primera sección, se administrarán las solicitudes, así como la asignación del personal que asistirá al AM, y mostrará la ubicación exacta a donde tendrá que acudir la persona asignada a dicha solicitud. Véase la Figura 3. La segunda sección llevará el control de los $\mathrm{AM}$, es decir, deben de estar registrado en el sistema de la aplicación web para poder tener información puntual del AM. Cabe mencionar que cuando se indica "asignación del personal que asistirá al AM", se refiere a los recursos humanos voluntarios que ayudarán in-situ a los AM a realizar sus actividades cotidianas: Trasladarse con sus herramientas al lugar de trabajo o de regreso a casa, cargar cosas pesadas en su lugar de trabajo, realizar trabajo pesado, por mencionar algunas. Por ello en la aplicación web, se llevará un control de estas personas. Kui Xékua pretende de esta manera generar conciencia de la ayuda a este sector vulnerable en la comunidad, considerando que la suma de esfuerzos y rescate de valores, mejoran considerablemente el impacto de las TIC's en la gente que lo requiere.

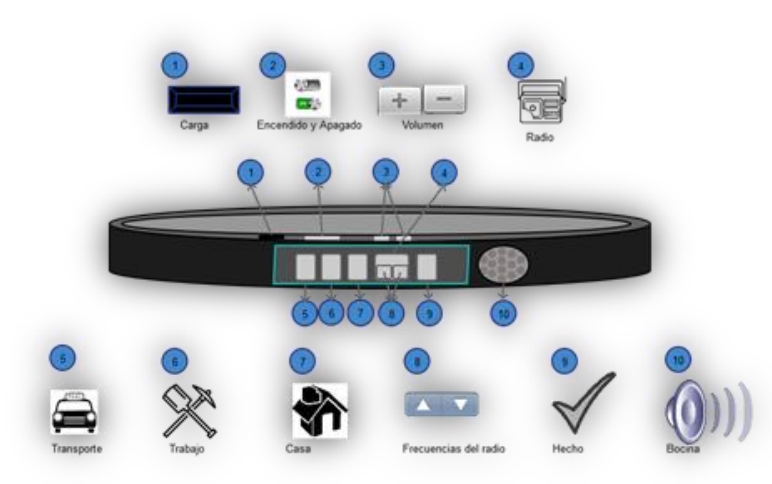

Figura 1. Brazalete (Wearable)

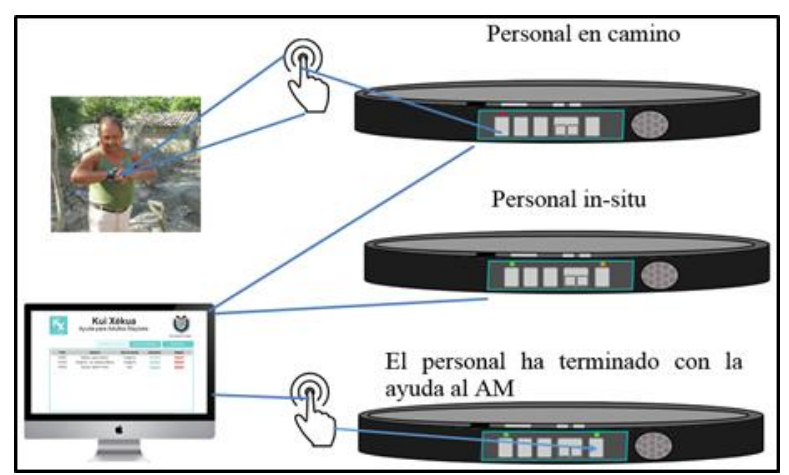

Figura 2. Solicitando ayuda desde el brazalete

\section{Metodología: UCD}

La metodología Diseño Centrado en el Usuario (por sus siglas en inglés, UCD) de Donald Norman comprende cinco fases [5], para el desarrollo de la presente propuesta se adaptó UCD a tres fases que se muestran en la Figura 4, debido a que es posible obtener un prototipo aceptable y evaluado.

\subsection{Fase de estudio}

Para comprender la situación en la que se encuentran los AM de la localidad de Teotitlán de Flores Magón, y las necesidades que se pudieran ver involucradas, se realizó un estudio contextual a través de entrevistas. En estas se desarrollaron preguntas, siendo los puntos de interés: las actividades que realizan en su vida cotidiana y el tipo de tecnología con el que interactúan.

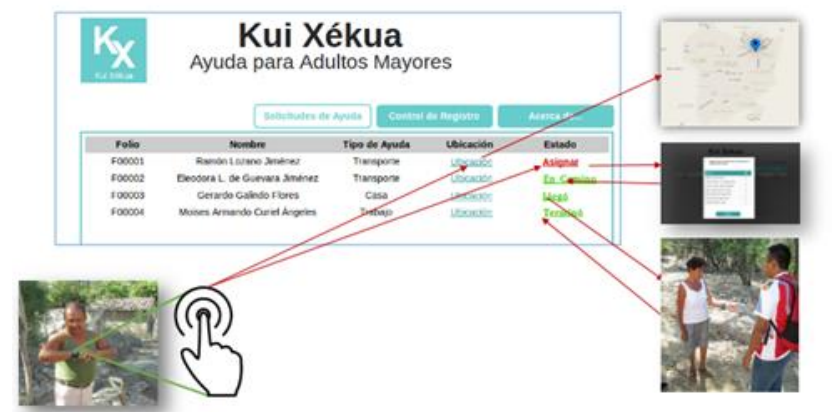

Figura 3. Atendiendo la solicitud de un AM desde la aplicación web

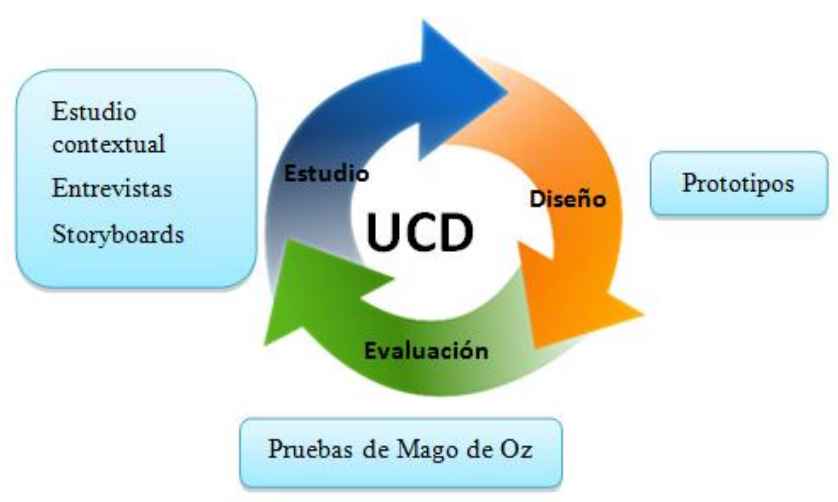

Figura 4. UCD

Analizando dicho estudio, se detectaron las necesidades de este grupo en específico: los AM requieren ayuda para realizar actividades cotidianas. Ellos a su edad siguen trabajando, porque consideran que aún pueden y deben hacerlo, es una forma de sentirse productivos. Sin embargo, indicaron que por su edad se ven reducidas algunas capacidades físicas. La Figura 5 muestra una gráfica que indica las actividades que les cuesta trabajo realizar: trabajos pesados, caminar y actividades domésticas. También los AM indicaron que suelen usar tecnología básica en casa como el televisor, estéreo, reproductor de DVD, radio, y el $50 \%$ de los entrevistados usan teléfonos celulares en su vida diaria. La mayor parte de las personas entrevistadas no usan la computadora. Los AM están dispuestos a aprender a usar el tipo de tecnología que se proponga para el beneficio propio. La Figura 5, también muestra el comportamiento del uso de la tecnología en los AM. Derivado de dicho estudio es que se consideraron las siguientes acciones: Es de suma importancia ayudar a estas personas, ya que su salud puede verse perjudicada al realizar una actividad física que precise un gran esfuerzo. La interacción entre las TIC's y los AM es escasa, sin embargo, es viable considerar la inclusión de las TIC's en la ayuda que se pueda brindar a los AM. En consecuencia, al estudio contextual, se desarrolló un storyboard (ver Figura 6), que contempla las primeras ideas de la solución propuesta del servicio Kui Xékua. El storyboard comprende tres secciones principales: "solicitar ayuda", "la ayuda 
se requiere en" y "ayuda in-situ". La primera es donde se considera la inclusión de las TIC's, mediante una aplicación web gestionada desde una central, y el brazalete que lo porta el AM, ambos estarían sincronizados. La segunda es el escenario del AM que se encuentra trabajando y en algún momento por las actividades a realizar va a requerir de apoyo, y con ayuda del brazalete lo solicita. Finalmente, la tercera es cuando la aplicación web detecta la ayuda, localiza al AM y envía a personal de apoyo. Con esto se lograría el objetivo: ayudar al AM en su actividad. Los elementos que conforman estas ideas, se detallarán en las siguientes secciones. Siguiendo al storyboard, se definieron los perfiles de usuarios de las personas que interactuarán con las TIC's en el servicio Kui Xékua. Se identifican como usuarios a los AM con el brazalete, y al administrador de la aplicación web, quien detecta la ayuda y la gestiona. La Tabla 1, detalla puntualmente los perfiles de usuarios.

\section{- ¿Qué actividades le cuesta trabajo realizar?}

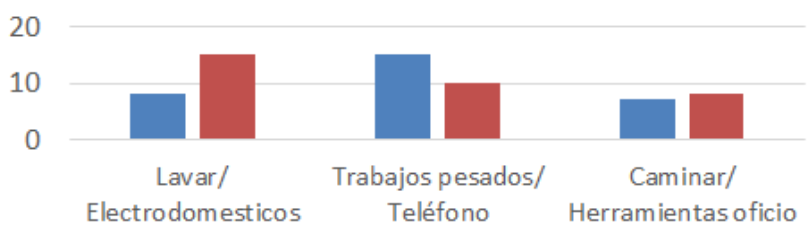

Figura 5. Actividades cotidianas
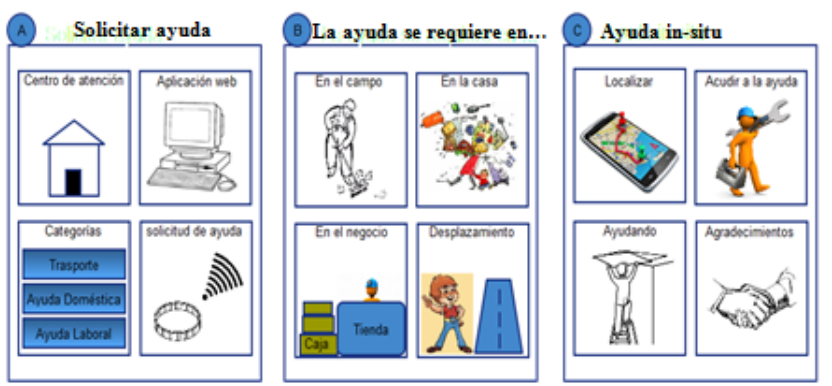

Figura 6. Storyboards

Tabla 1. Perfiles del usuario

\begin{tabular}{|l|l|l|}
\hline & Adultos mayores & $\begin{array}{l}\text { Administrador de la } \\
\text { aplicación web }\end{array}$ \\
\hline Género & \multicolumn{2}{|c|}{ Femenino y masculino } \\
\hline Edad & De 55 años a 80 años & De 20 a 35 años \\
\hline Escolaridad & $\begin{array}{l}\text { Nivel básico, medio } \\
\text { superior y superior }\end{array}$ & $\begin{array}{l}\text { Medio superior y } \\
\text { superior }\end{array}$ \\
\hline Idioma & Español y mazateco & Español \\
\hline Nacionalidad & Mexicana & Mexicana \\
\hline
\end{tabular}

Con esta fase se ha detallado el contexto de los usuarios, los perfiles de los usuarios, aspectos esenciales para el desarrollo de un producto, servicio, o artefacto, bajo el esquema de UCD.

\subsection{Fase de diseño}

A partir de la fase anterior se realizó el diseño de Kui Xékua, una solución centrada en el usuario, que busca ayudar a los AM. Para el desarrollo del diseño de Kui Xékua, se optó por el uso de prototipos de baja fidelidad. A continuación, se presenta las versiones de las herramientas que comprenden el servicio: el brazalete y la aplicación web.

\subsubsection{Diseño de la aplicación web}

La aplicación web Kui Xékua fue evolucionando en sus versiones prototipo (ver Figura 7). La navegación mejoró de vertical a horizontal, y en algunas secciones, se consideraron ambas. Las tareas a realizar por el administrador web determinó el orden de las secciones de la aplicación. La consistencia en los colores, botones y formularios, se corrigieron. La retroalimentación para los usuarios mejoró en permitir que cuando realizara las tareas, le indicaría el estado de la información. Debido que es una versión prototipo no funcional, estos aspectos son los que fue posible abarcar, y con apoyo de las pruebas de Mago de Oz realizadas.

Prototipo aplicación 1.0

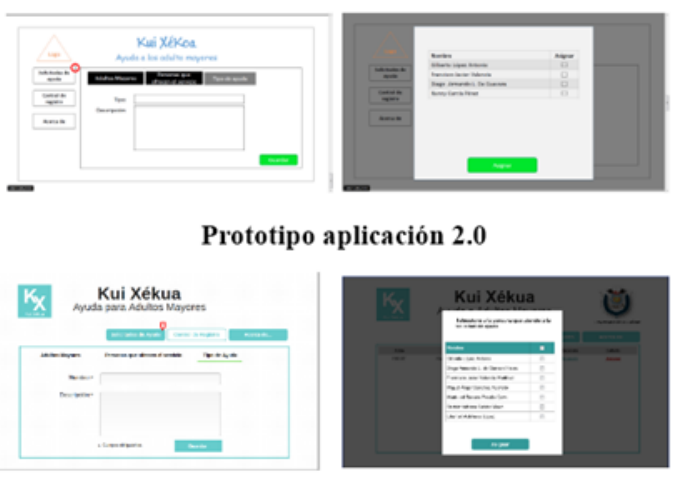

Figura 7. Aplicación web

\subsubsection{Diseño del brazalete}

Para el diseño del brazalete, se inicia asociando las tareas a realizar por el AM, con el orden de los elementos que lo conformarían. La iconografía que se utiliza son imágenes que relacionan los usuarios con las actividades en su trabajo cotidiano. El manejo de estados de las acciones en el brazalete es considerando la analogía de algunos dispositivos, cuando indican la carga de estos, es decir, se asocian los colores rojo, naranja y verde, a los estados. Se incluye la radio, como una acción adicional, que pueda acrecentar la experiencia de uso del brazalete, además que ellos indicaron que es una actividad que les gusta realizar mientras trabajan. La inclusión de retroalimentación a través del sonido, es un factor de apoyo para los AM, ya que, en la etapa de aprendizaje de los controles, y considerando aspectos cognitivos de su edad, beneficia este tipo de acción. La distribución de los componentes y tamaño del brazalete evolucionaron con apoyo de las pruebas de Mago de Oz. Ver Figura 8.

\subsection{Fase de evaluación}

Para verificar el entendimiento, facilidad de uso y encontrar mejoras del servicio Kui Xékua se realizaron pruebas de Mago de $\mathrm{Oz}$ [6]. Debido a que el servicio consiste en dos herramientas, en las siguientes secciones se detallan los resultados para cada una.

\subsubsection{Brazalete}

Se realizaron 15 pruebas de Mago de Oz con los AM. Las pruebas se basaron en llevar el brazalete construido a los usuarios y simular su funcionamiento, que daba la apariencia real del dispositivo. La selección de los usuarios fue con base al perfil del usuario para el grupo de los AM. Las pruebas se realizaron en el 
lugar de trabajo del usuario. Dichas pruebas incluían las siguientes tareas: solicitar ayuda de traslado, solicitar ayuda en el trabajo, solicitar ayuda en el hogar e identificar los componentes del brazalete. En esta evaluación los usuarios interactuaron correctamente con el brazalete, realizaron las tareas de manera eficiente y mostraron su satisfacción, sobre todo por la retroalimentación que les proporcionaba el brazalete, con el audio y el manejo de colores en los diferentes estados en los que se encontraban las opciones. Además, que la iconografía utilizada en los botones del brazalete se identificó correctamente en su mayoría. El problema común al que se enfrentaron fue con el botón "hecho" y "radio". Sin embargo, después de oprimirlos, y se simulara su acción, para la segunda tarea fue más efectivo el uso de estos botones.

Prototipo Brazalete 1.0

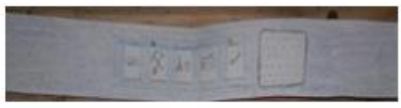

Prototipo Brazalete 2.0

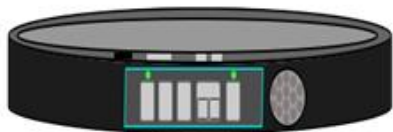

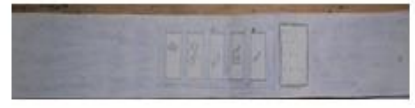

Prototipo Brazalete 3.0

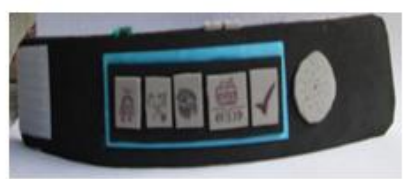

Figura 8. Brazalete

Como parte importante del estudio, fue explorar las experiencias y/o emociones con el uso del brazalete a los AM, por lo que ellos listaron lo siguiente: admiran aún más la tecnología, curiosidad por interactuar, se sienten útiles y que pueden seguir adelante en sus actividades (los empodera), expectativa sobre el brazalete real.

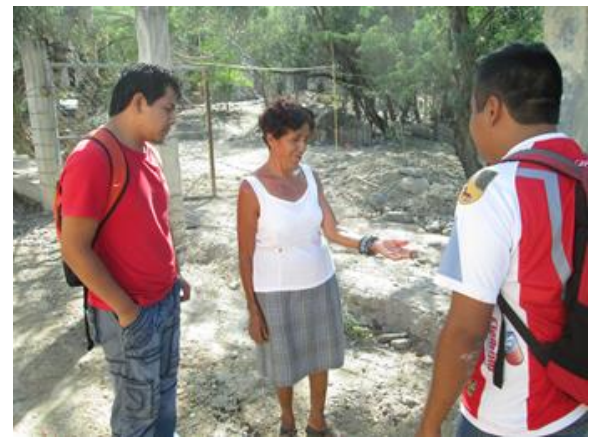

Figura 9. Mago de Oz, Wearable

\subsubsection{Aplicación web (AW)}

Se realizaron seis pruebas de Mago de $\mathrm{Oz}$ con los usuarios con perfil de administrador de la AW. Las pruebas se basaron en capturas de pantalla (prototipos en papel) que dan la apariencia real del sistema. La selección de los usuarios fue con base al perfil del usuario para este tipo de usuarios. Las pruebas se realizaron en una casa particular, que fue el punto de reunión para los usuarios. Dichas pruebas incluían las siguientes tareas: registrar a un AM en la aplicación, registrar al personal de apoyo para brindar la ayuda al AM, atender la solicitud de ayuda del AM. En esta evaluación los usuarios interactuaron correctamente con la aplicación, realizaron las tareas de manera eficiente y mostraron su satisfacción. El problema común al que se enfrentaron fue confusión en el nombrado de los menús principales de la AW, y que algunas acciones como la "localización de los AM", tuvieran más retroalimentación. Como parte importante del estudio, fue explorar las experiencias y/o emociones con el uso de la AW, si bien los usuarios ya cuentan con experiencia en el manejo de este tipo de aplicaciones, lo más relevante fue que esta sería una aplicación que se comunicaría con un brazalete y mejor aún, por el servicio que ofrecería, por lo que sus emociones fueron interés y expectativa.

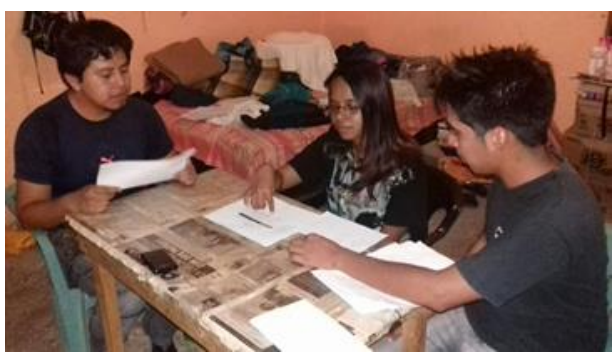

Figura 10. Mago de Oz, aplicación web

\section{Conclusiones}

Kui Xékua como servicio, pretende coadyuvar en la mejora de la calidad de vida de los AM, permitiéndoles que formen parte de la sociedad económicamente activa y permite seguir empoderándolos con apoyo de otras personas y de la tecnología existente. Esta solución acrecentará el uso de la tecnología en los AM, y puede extender su funcionalidad a otros aspectos vulnerables a su edad, como la salud, seguridad y monitoreo de los AM. Kui Xékua ofrece en todo momento retroalimentación de su servicio a los AM. Kui Xékua práctica la ayuda entre personas, impactando en los valores humanos de la sociedad, para apoyar a los sectores vulnerables.

\section{Agradecimientos}

Agradecemos a los AM de la localidad de Teotitlán de Flores Magón, por su participación como usuarios con Kui Xékua. También agradecemos a los estudiantes universitarios de la población por su colaboración como usuarios de la aplicación web.

\section{Referencias}

[1] MexIHC. 2016. Student Design Competition. MexIHC 2016. Sitio web: http://www.mexihc.org/2016/sdc.html

[2] Noel Torres. 2015. Datos de Proyecciones. SEGOB, CONAPO Sitio web: http://www.conapo.gob.mx/es/CONAPO/Proyecciones_Dato $\mathrm{s}$

[3] Rivera de Peña, Angélica; Vargas Carrillo, Laura. 2010. Desarrollo Integral de la Familia. DIF Nacional. Sitio web: http://sn.dif.gob.mx/

[4] Borja, Robert. 2014. Tecnologías emergentes. ABCTecnología. Sitio web: http://www.abc.es/tecnologia/informaticahardware/20140107/wearables-complementos201401071108.html

[5] Norman, D. y Draper, S. 1986. User Centered System Design: New Perspectives on Human-Computer Interaction. L. Erlbaum Associates Inc. Hillsdale, NJ, USA.

[6] Usability Net. 2006. Wizard of Oz. Usability Net. Sitio web: http://www.usabilitynet.org/tools/wizar 